\title{
Design and Simulation of Capacitive MEMS Switch for Ka Band Application
}

\author{
Vinay Bhatia $\mathbb{D}^{1},{ }^{1}$ Sukhdeep Kaur ${ }^{\mathbb{D}},{ }^{1}$ Kuldeep Sharma ${ }^{(D)},{ }^{1}$ Punam Rattan ${ }^{(D)}{ }^{2}$ \\ Vishal Jagota $(i),{ }^{3}$ and Mohammed Abdella Kemal $\oplus^{4}$ \\ ${ }^{1}$ Department of Electronics and Communication Engineering, CGC-CEC Landran, Punjab, India \\ ${ }^{2}$ Punjab Technical University, Punjab, India \\ ${ }^{3}$ Department of Mechanical Engineering, Madanapalle Institute of Technology \& Science, AP, India \\ ${ }^{4}$ Arba Minch University, Ethiopia
}

Correspondence should be addressed to Mohammed Abdella Kemal; abdella.kemal@amu.edu.et

Received 13 May 2021; Revised 13 June 2021; Accepted 29 June 2021; Published 13 July 2021

Academic Editor: Vimal Shanmuganathan

Copyright (C) 2021 Vinay Bhatia et al. This is an open access article distributed under the Creative Commons Attribution License, which permits unrestricted use, distribution, and reproduction in any medium, provided the original work is properly cited.

In this paper, RF MEMS switch with capacitive contact is designed and analyzed for Ka band application. A fixed-fixed beam/meander configuration has been used to design the switch for frequency band $10 \mathrm{GHz}$ to $40 \mathrm{GHz}$. Electromagnetic and electromechanical analysis of three-dimensional (3D) structure/design has been analyzed in multiple finite element method (FEM) based full-wave simulator (Coventorware and high-frequency structure simulator). A comparative study has also been carried out in this work. The high resistivity silicon substrate $\left(\tan \delta=0.010, \rho>8 \mathrm{k} \Omega-\mathrm{cm}, \varepsilon_{r}=11.8\right)$ with a thickness of $675 \pm 25 \mu \mathrm{m}$ has been taken for switch realization. The designed structure shows an actuation voltage of around $9.2 \mathrm{~V}$. Impedance matching for the switch structure is well below $20 \mathrm{~dB}$, loss in upstate, i.e., insertion loss $>0.5 \mathrm{~dB}$, and isolation of $>25 \mathrm{~dB}$ throughout the frequency band is observed for the aforesaid structure. Furthermore, to increase the RF parameters, AIN dielectric material has been used instead of $\mathrm{SiO}_{2}$ resulting in capacitance in downstate that increases hence improved the isolation. The proposed switch can be utilized in various potential applications such as any switching/tunable networks phased-array radar, reconfigurable antenna, RF phase shifter, mixer, biomedical, filter, and any transmitter/receiver (T/R) modules.

\section{Introduction}

The Micro-Electro-Mechanical-System (MEMS) is an agglomeration of submicron mechanical moving parts and electronics components/elements on a single module/chip [1]. In the last 20 years, MEMS has spread into the RF regime also. RF MEMS switches are building blocks for this technology, without the existence of these switches; RF MEMS seems to be unimaginable $[1,2]$. Nowadays, RF MEMS capacitive shunt switch has a major role for designing in an advanced communication application. RF MEMS switches are preferable to design filters, reconfigurable microwave antenna, and space communication than solid-state switch like fieldeffect transistor (FET) and PIN diode. RF MEMS switches based on MEMS technology offer many advantageous over solid-state switches, for example, high isolation, low insertion loss, zero DC power consumption, low noise, more linearity, and used up to millimeter wave [2-4]. MEMS technologybased RF switches have the advantages of miniaturization, monolithically integrated with other IC using batch processing technology as compared to other conventional switches. The performance of the RF MEMS shunt switch depends upon the material used for the membrane and also depends upon return loss, pull-in voltage, insertion loss, isolation, and switching time [1]. Metal-metal contact in the switch creates the stiction problem in the switch. Based on MEMS technology, RF MEMS switches have two types such as shunt switch and series switch [3]. These series and shunt configuration further classified as a capacitive switch and resistive switch base upon contact [2]. Series switches are used for a 
low-frequency application due to poor isolation, while for a higher frequency of application, capacitive switches are used. In capacitive type contact MEMS switch, a dielectric layer is used between the top beam and bottom electrodes for isolation. The structure of the membrane can be bridge type or cantilever type or dip harm type [3-6]. The actuation voltage of the switch structure can be minimized with bridge structure supporting meanders and step type [1-4]. MEMS-based switches are superior in terms of their RF performances over conventional electromagnetic relays and solid-state switches [5-8]. As per the configuration, the switch can be broadly classified into two categories, i.e., series and shunt $[1,2]$. Generally, series switch utilizes metal-metal contact, while shunt switches are mostly capacitive in nature [9]. Some sort of actuation force is needed to make these switches work. Although several actuation mechanisms are there, the electrostatic actuation technique is extensively used due to its negligible DC power consumption, short switching time, and compatibility with IC technology $[1,2,10]$. The reliability of space systems is of utmost importance for their smooth operation over a period of 10-15 years of satellite life. A single point failure may cause catastrophe for the whole mission. So, each and every component, be in launch vehicle or communication systems need to be highly reliable $[8,11,12]$. Metal contact reliability in series switches has always been a concern during long-term reliability tests [13]. A switch based on metal-metal contact normally suffers from various issues like electromigration, stiction, metal creep, dielectric charging, and fatigue over a period of time $[12,14]$. Stiction issue in MEMS switch occurs, when surface adhesive forces are larger than the restoring force of the beam structure [15]. Electromigration is a high current density phenomenon which causes voids in metal lines. While creep describes the trends of a material to deform or to move permanently to relieve stresses, resulting in a long-term revelation to levels of stresses due to material deformation, which are below the yield or final strength of the material $[8,14]$. Keeping in mind all the abovementioned issues associated with metal contact switches, capacitive contact switch was taken into consideration [8]. A MEMS capacitive shunt switch serves as a two-state digital capacitor: a smaller capacitance $(\mathrm{fF})$ is obtained while the switch is in up position, while a larger capacitance value of capacitor $(\mathrm{pF})$ results in downstate of the switch $[1,2]$. Generally, RF MEMS capacitive switch uses silicon nitride as dielectric layer due to its medium dielectric constant $\left(\varepsilon_{r}=7.5\right)$, which provides the isolation between both electrodes [16]. Other dielectric material such as $\mathrm{TiO}_{2}\left(\varepsilon_{r}=32\right)$, strontium titanate oxide $\left(\varepsilon_{r}=120\right.$ [16]), hafnium dioxide $\left(\mathrm{HfO}_{2}, \varepsilon_{r}=25\right.$ [17]), piezoelectric lead zirconate titanate (PZT, $\varepsilon_{r}=190$ [18]), and aluminium nitride (AIN, $\varepsilon_{r}=9.8$ [19]) can also be utilized because of high dielectric constant in place of silicon nitride layer for impressive RF performance.

Many researchers and industries around the globe have reported various RF MEMS switches using different materials with various technologies and by varying the electromechanical and RF parameters. Somayye et al. developed the shunt switch with reduced air gap using step structure, and AIN dielectric layer is used instead of $\mathrm{SiO}_{2}$ and $\mathrm{Si}_{3} \mathrm{~N}_{4}$ for

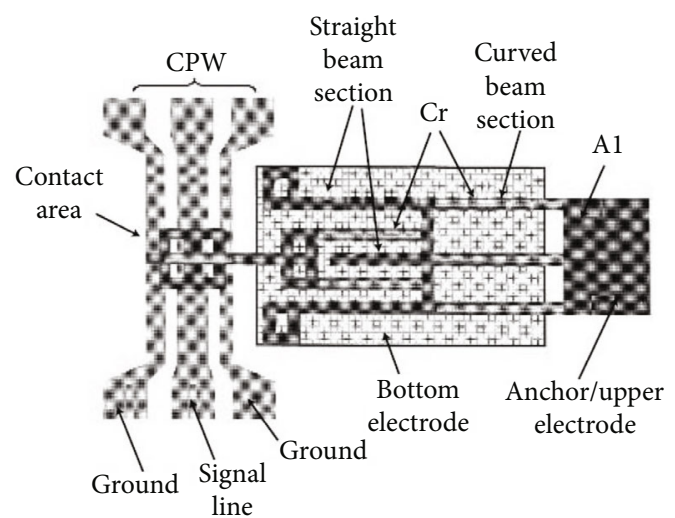

Figure 1: Fabricate capacitive shunt switch National Taiwan University [1].

improved isolation, i.e., $-58 \mathrm{~dB}$. Chan et al. have developed the DC contact switch using GaAs substrate at National Taiwan University as shown in Figure 1. In this switch, the coplanar waveguide (CPW) line and center conductor are fabricated using gold material and $\mathrm{SiO}_{2}$ was used as a dielectric layer. The authors reported the return loss $>20 \mathrm{~dB}$, isolation of $>20 \mathrm{~dB}$, and actuation voltage around 26-30 V [15-23].

Furthermore, Guo et al. [24] developed the low actuation voltage MEMS switch by utilizing aluminum alloys for movable beam. The designed switch shows the low pull-in voltage of $5 \mathrm{~V}$ for alloy instead of gold material, i.e., $45 \mathrm{~V}$. Rao et al. fabricate the MEMS switch using the clamped-clamped structure of gold beam having thickness $500 \mathrm{~A}^{0}$, and the dielectric layer of AIN is deposited by plasma-enhanced chemical vapor deposition (PECVD) process. The authors reported the actuation voltage of $5.5 \mathrm{~V}$ and behaviors of dielectric charging to characterize by the capacitance-voltage (CV) curve method. Goel et al. developed the MEMS series switch having low actuation voltage, improved RF performance for reconfigurable antenna, and sufficient switching speed. The authors reported the pull-in voltage of $5 \mathrm{~V}$ with return loss $>20 \mathrm{~dB}$ and insertion loss $>0.4 \mathrm{~dB}$ from $\mathrm{DC}$ to $60 \mathrm{GHz}$. Further, the capacitive shunt switch was fabricated on a four-mask silicon wafer by utilizing surface micromachining process technology to report the low actuation voltage. The PECVD is used to deposit a thin AIN dielectric layer to improve isolation in the downstate due to a high dielectric constant. Therefore, Thelluir et al. developed the low pull-in voltage MEMS switch using gold as a beam material with improved RF performance. Chu et al. fabricate the MEMS switch on GaAs substrate using a pull-in electrode and inline cantilever beam to achieve "On" and "Off" states as shown in Figure 2. In this train, spaced holes are etched into cantilever beam to achieve the lower pull-in voltage. The measured insertion loss of $>3 \mathrm{~dB}$ and isolation of $>20 \mathrm{~dB}$ in the frequency range $1-40 \mathrm{GHz}$ was reported.

\section{RF Actuation Mechanism}

Mechanical actuation and electrical actuation are two parts of the RF MEMS switch. Mechanical movement can be realized 


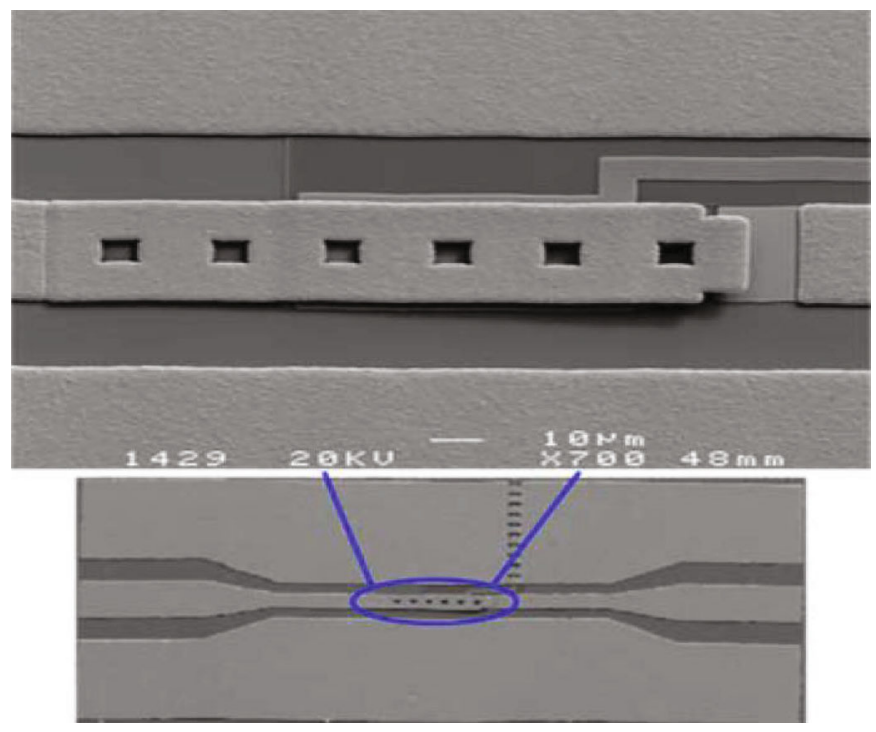

FIGURE 2: Scanning electron microscope image of DC contact switch developed by Chu et al. [17].

TABLE 1: Different actuation techniques for MEMS switch.

\begin{tabular}{lcccccc}
\hline Technology & Contact force $(\mu \mathrm{N})$ & Current $(\mathrm{mA})$ & Size & Voltage $(\mathrm{V})$ & Switching time $(\mu \mathrm{s})$ & Power $(\mathrm{mW})$ \\
\hline Magnetostatic & $50-200$ & $20-150$ & Medium & $3-5$ & $400-1000$ & $0-100$ \\
Thermal & $500-3500$ & $5-100$ & Large & $3-5$ & $200-10,000$ & $0-200$ \\
Piezoelectric & $50-250$ & 0 & Medium & $3-20$ & $50-500$ & 0 \\
Electrostatic & $50-1500$ & 0 & Small & $20-80$ & $1-200$ & 0 \\
\hline
\end{tabular}

by piezoelectric, electromagnetic, electrostatic, and thermal mechanisms. The common approach used now days is electrostatic actuation mechanism, due to its advantages like low DC power consumption, low switching speed, thin layers of material, wide range of contact forces of $50-200 \mu \mathrm{N}$, and most importantly tiny electrode size. Its ON-OFF capacitance ratio is also very high, but a higher actuation voltage is required. In comparison to electromagnetic actuation, voltage requirement is low but current consumption is high. Coil and ferromagnetic armature are used as an electromagnetic actuator in electromagnetic actuation. In this type of mechanism, semihard magnetic materials or permanent magnets allow the addition of a self-latching mechanism. The example of MEMS device that uses this mechanism is a micromechanical relay. A thermal actuator can also be used to simplify the relay. Thermal actuators include the armature of a soft magnetic material, permanent magnets, and thermosensitive magnetic materials stators. The piezoelectric mechanism depends upon the piezoelectric effect, and it provides exceptional material characteristics, where elastic deformation is generated by electrical field stimulation. To actuate $\mathrm{ON}$ or OFF conditions, a downward force is created by attaching a designed piezoelectric actuator to switch the membrane. Reduced pull-in voltage may be obtained by enabling the switch to have a high force by using this mechanism. This high force permits the air gap height to be increased with no reduction in another parameter of the switch such as critical stress and switching speed. The switch can also be actuated by using a thermal actuation mechanism. This actuation mechanism includes the thermal microactuators. For example, two thick metal posts are linked with the two thermal actuators which act as the centerline of the CPW transmission line, and the MEMS switch may be trigger $\mathrm{ON}$ and OFF by utilizing current or voltage pulses. A very few researchers had been developed the MMES switch using thermal actuation with a huge contact force and large beam spring constant to achieve the low contact resistance and hence enhance the power handling capacity. Table 1 shows the comparisons between four actuation technologies, which are used to actuate the RF MEMS switch.

\section{Design and Working Principal}

A high resistive silicon wafer (thickness of $675 \pm 20 \mu \mathrm{m}$, dielectric constant and 11.8) has been used for the development of MEMS switch using fixed-fixed beam configuration. Figures 3(a) and 3(b) show the cross-section view of the proposed MEMS capacitive switch in upstate position and downstate position, respectively. The coplanar waveguide transmission line with dimension (G/W/G) of 90/120/90 (all in $\mu \mathrm{m}$ ) is designed to support the RF MEMS switch whose characteristics impedance is $50 \Omega$. The grounds of the CPW line are connected to both ends of the MEMS 


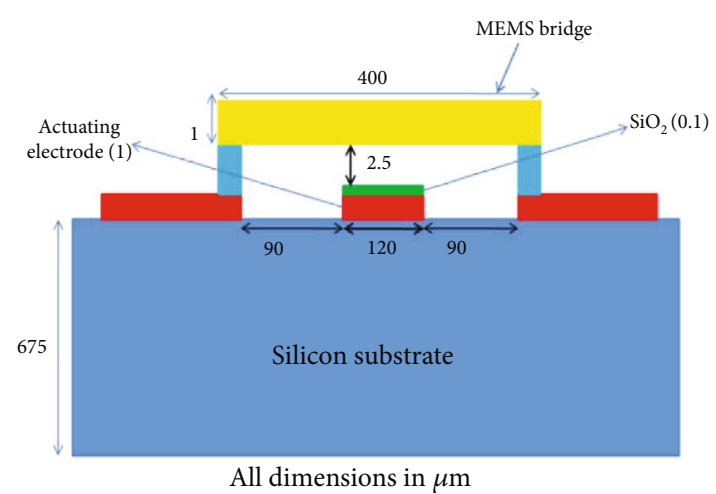

(a)

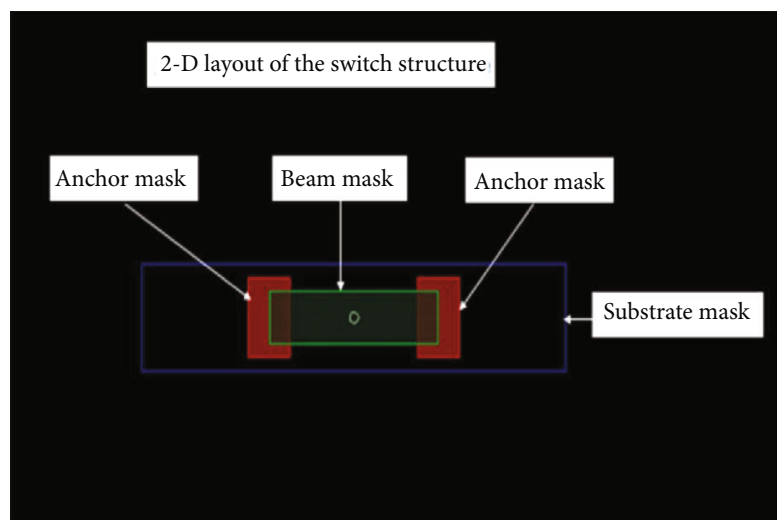

(c)

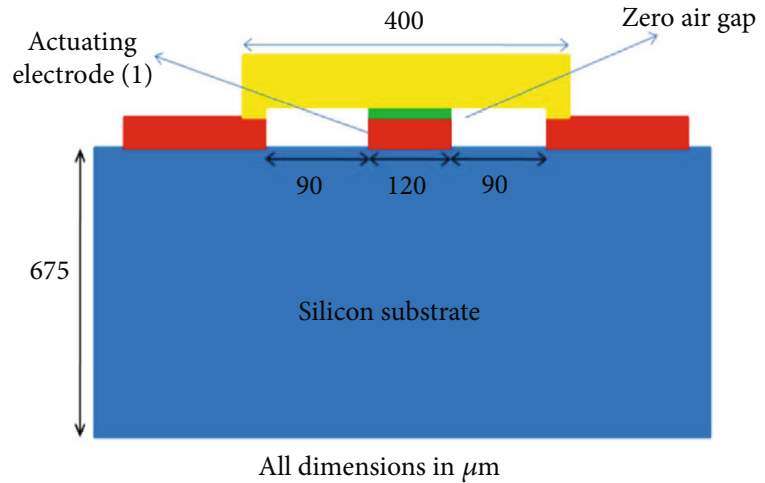

(b)

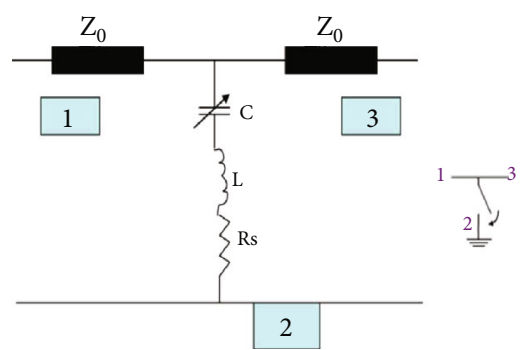

(d)

FIGURE 3: (a) Cross-section view of MEMS capacitive switch in upstate (b) in downstate position. (c) 2D layout of switch structure in Coventorware. (d) Electrical equivalent model of switch in downstate.

switch through the anchors. The material used for anchors, transmissions line, and bridge is gold because resistivity is higher than other conducting materials such as aluminum, silver, and copper. A thin dielectric layer is used above the center conductor transmission line, which is used to prevent the upper electrode and bottom electrode from short-circuit. The dielectric materials between both conducting electrodes are chosen in such a way that having high relatively permittivity results in the low losses and lower pull-in voltage. The proposed switch has been simulated using $\mathrm{SiO}_{2}$ and AIN dielectric materials to study the impact on the RF performance of the switch.

The electrical actuation and mechanical actuation are two sections of the RF MES switch. To actuate the mechanical section, there are four types of actuation mechanism, i.e., piezoelectric, electrostatic, thermal, and electromagnetic mechanisms. The common approach used nowadays is the electrostatic actuation mechanism.

When there is no DC voltage applied between the beam and bottom electrode, the switch is in ON state and signal pass from one port to another port. On the other hand, when the DC voltage is applied between them, the switch is in the OFF state. Hence, membrane collapses on the dielectric layer which is above the center transmission. Due to electrostatics force between top and movable beam the signal pass from one port to another port blocks. This electrostatic force is caused by actuation voltage applied between both electrodes and is given as $[1,2]$.

$$
F=\frac{\varepsilon_{0} w W V_{a}^{2}}{2\left[g_{0}+\left(t_{d} / \varepsilon_{r}\right)\right]^{2}},
$$

where $V_{a}$ is actuation voltage and is given by [1].

$$
V_{a}=\sqrt{\frac{2 k g^{2}\left(g_{0}-g\right)}{\varepsilon_{0} w W}},
$$

where $W$ is the width of the CPW transmission line, $g_{0}$ and $g$ are pre- and postair gap height from membrane, $w$ is the beam width, and $k$ is the spring constant of the beam in negative $z$ direction and is given as $[1,5]$

$$
\begin{aligned}
& K=K^{\prime}+K^{\prime \prime}, \\
& K=32 E w(t / l)^{3}(27 / 49)+8 \sigma(1-v) w(t / l),
\end{aligned}
$$

where $E(\mathrm{GPa})$ is Young's modulus, $\sigma(\mathrm{MPa})$ is biaxial residual stress due to fabrication process, $K^{\prime}$ is due to the stiffness of structure, $K^{\prime \prime}$ is spring constant contribution due to residual stress, and $v$ is Poisson's ratio of beam. The detailed 
dimension and material used for the switch structure are given in Table 2.

Above a definite threshold voltage, the electrostatic force is increased due to an increase in charge and overcome the mechanical restoring force of the beam. An increase in electrostatic force results in a decrease in the beam gap height and at the same time which in turn enhances the downstate capacitance, and resulting beam becomes unstable and this threshold voltage is called pull-in voltage given as $[1,14]$

$$
V_{p}=V\left(2 g_{0} / 3\right)=\sqrt{\frac{8 k g_{0}^{3}}{27 \varepsilon_{0} w W}}
$$

\section{Electrical Model of Shunt Switch}

The electrical equivalent model describes a shunt switch as CLR (capacitance, inductance, resistance) series resonant circuit as shown in Figure 3(d). The movable metallic beam of the MEMS shunt switch is sketched by switch variable capacitance, lumped resistance, and switch inductance. Depending upon the ON and OFF conditions of the switch, the variable capacitance is changed. When the switch in ON condition, the variable capacitance is represented by $C_{\text {up }}$ and in OFF condition it is represent by $C_{\text {down }}$. From Eq. (6), if the frequency of operation is less than LC resonance frequency, the CLR model works as a capacitor, while it works as an inductor above LC resonant frequency. At the series LC resonant frequency, the CLR (capacitance, inductance, resistance) model reduces as series resistance and is represented by $R_{s}$. Capacitances in up- and downstate define the insertion and isolation of shunt switch, respectively. The following subsections explain these points elaborately. The impedance of the capacitive shunt switch is given as [5]

$$
\begin{aligned}
& Z_{s}=R_{s}+j \omega L+\frac{1}{j \omega C}, \\
& Z_{s}=\left\{\begin{array}{cc}
\frac{1}{j \omega C} & \text { for } f<<f_{0} \\
R_{s} & \text { for } f=f_{0} \\
j \omega L & \text { for } f>>f_{0}
\end{array}\right\},
\end{aligned}
$$

where $C$ is the capacitance of the switch it may be up capacitance $\left(C_{u}\right)$ and down capacitance $\left(C_{d}\right)$ depends upon the switch position, $R_{s}$ is the bridge resistance and it depends upon the resistivity of bridge, and $L$ is the bridge inductance and it depends upon the gap between signal line ground of CPW. The LC series resonant frequency of the capacitive shunt MEMS switch is given as [1]

$$
f_{0}=\frac{1}{2 \pi \sqrt{L C_{d} / C_{u}}} .
$$

\section{Result and Discussion}

5.1. Pull-In Analysis. The modal analysis and pull-in voltage for fixed-fixed beam has been carried using the finite element
TABle 2: Specific parameters of the proposed MEMS switch.

\begin{tabular}{lc}
\hline Parameters & Value \\
\hline Beam length $(L)$ & $400 \mu \mathrm{m}$ \\
Beam width $(w)$ & $120 \mu \mathrm{m}$ \\
Center conductor width $(W)$ & $120 \mu \mathrm{m}$ \\
Overlap area $(A)$ & $120 \times 120=14400 \mu \mathrm{m}^{2}$ \\
Dielectric material and constant & AIN \&11 \\
Beam thickness $(t)$ & $1 \mu \mathrm{m}$ \\
Beam material & Gold \\
Dielectric thickness $\left(t_{d}\right)$ & $0.1 \mu \mathrm{m}$ \\
Air-gap $\left(g_{0}\right)$ & $2.5 \mu \mathrm{m}$ \\
Substrate thickness $(h)$ & $675 \pm 20 \mu \mathrm{m}$ \\
CPW dimension & G/W/G- $90 \mu \mathrm{m} / 120 \mu \mathrm{m} / 90 \mu \mathrm{m}$ \\
CPW thickness & $1 \mu \mathrm{m}$ \\
\hline
\end{tabular}

method- (FEM-) based Coventorware software. At the pullin voltage, the movable bridge/membrane suddenly collapses on the thin dielectric layer due to the mechanical action of electrostatic force and causing the system to unstable (Figure 4). The electromechanical analyses in Coventorware are shown in Figure 5. It can be seen from the plot as voltage increases on the center conductor the top movable metallic beam starts to snap down from the top in the negative $z$ direction towards the dielectric layer due to electrostatic force. When the metallic beam travel $1 / 3^{\text {rd }}$ gap of the total initial gap from the topside, the movable beam enters into the instable region/area, and at this condition, electrostatic force overcome the restoring force. Small diameters $\left(10 \times 10 \mu \mathrm{m}^{2}\right)$ holes are introduced in the top metal membrane to reduce the stiction problem and residual stress during the release process as shown in Figure 4.

5.2. Switching Time. The switching time depends upon the mechanical resonant frequency of the movable beam, applied source voltage, and pull-in voltage. It is defined as a time taken by the movable metallic beam to toggle from actuated state to unactuated state and vice versa is called switch speed and is given as [1].

$$
T_{s}=3.67 \frac{V_{p}}{V_{s} \omega_{0}}
$$

where $V_{s}$ is the source voltage and given by $V_{s}=1.35 V_{p}, \omega_{0}$ is the mechanical resonant frequency and given by as $\omega_{0}=$ $\sqrt{k / m}, m$ is the beam mass, and $k$ is the spring constant in negative $z$ direction. Figure 6 demonstrates the variation of switching speed/time with beam thickness to beam length $(t / l)$ ratio. The switching in gold membrane is three times slower compared to aluminum.

5.3. RF Analysis. The ANSYS HFSS software has been used to extract the RF performance of the designed fixed-fixed beam configuration-based MEMS switch. RF parameters such as insertion loss $\left(S_{21}\right.$ in $\left.\mathrm{dB}\right)$ and return loss $\left(S_{11}\right.$ in $\left.\mathrm{dB}\right)$ are defined in upstate, whereas isolation $\left(S_{21}\right.$ in $\left.\mathrm{dB}\right)$ is defined 


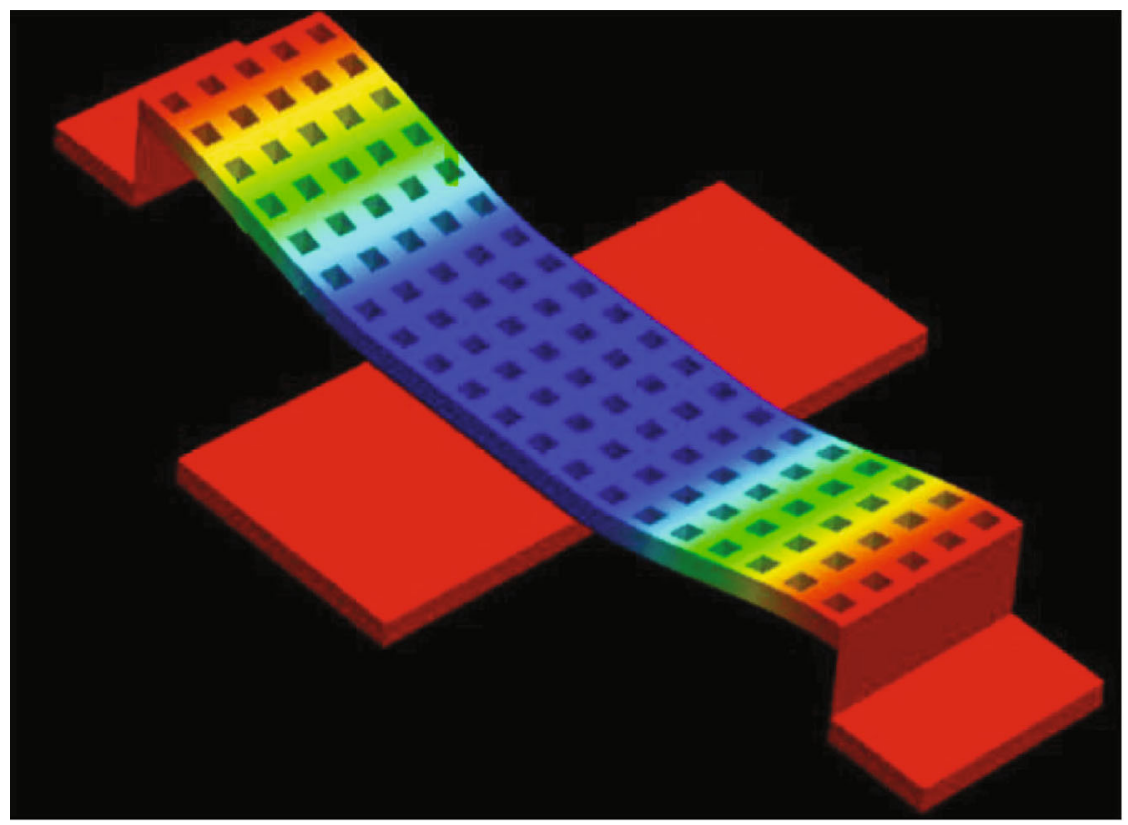

Figure 4: 3D view of proposed switch in Coventorware.

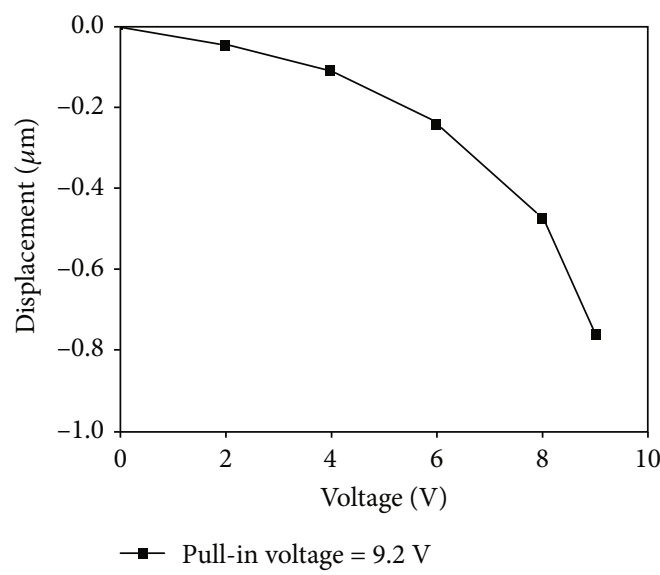

Figures 5: Variation of initial gap with applied voltage.

in downstate. When there is no voltage applied to the bottom electrode, the switch is in unactuated state, and in this state, two RF parameters such as return loss and insertion loss of the switch have been analyzed. Return loss state that how much power is reflected back by the input/exciting port of coplanar waveguide transmission line. This is due to the improper impedance matching between switch and CPW line. Figure 7 shows the return loss or impedance matching as a function of frequency is better than $20 \mathrm{~dB}$ over whole frequency band. The return loss $\left(S_{11}\right)$ in the up state of the switch is mathematically calculated by [1]

$$
\left|S_{11}\right|^{2} \approx \frac{\omega_{0}^{2} C_{u p}^{2} Z_{0}^{2}}{4} .
$$

Insertion loss is also calculated in upstate and is it change the flow of RF signal between the input port and output port

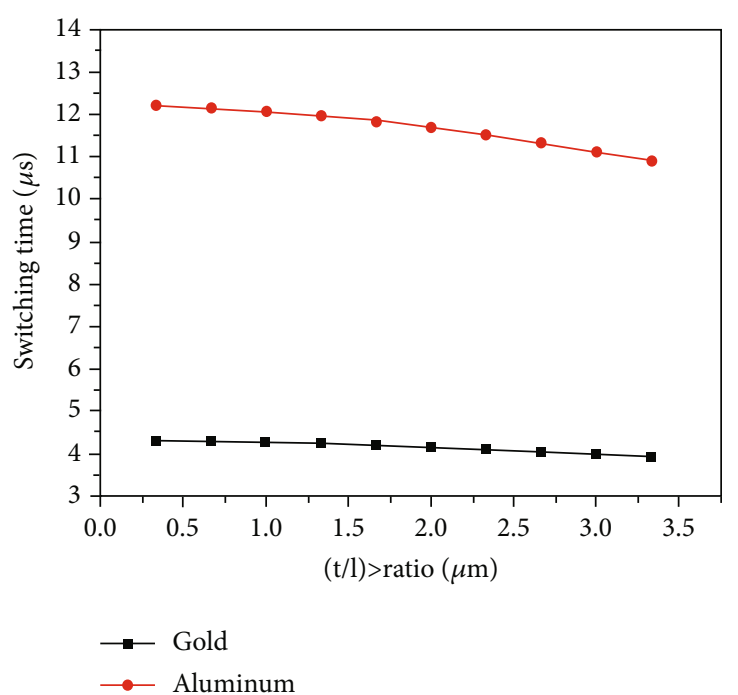

Figure 6: Variation of switching time $\left(t_{s}\right)$ with $(t / l)$ ratio of RF-MEMS Shunt Switch.

to control the power dissipations in transmission line and is given as [1].

$$
\left|S_{21}\right|=\left(\frac{1}{1+j \omega_{0} C_{d} Z_{0} / 2}\right)
$$

Insertion loss happens at low frequency due to the resistive loss between contact resistance region of the switch and finite resistance of CPW line while at high frequency is due to skin depth. Figure 8 shows the insertion loss better than $0.5 \mathrm{~dB}$ throughout the frequency band. 


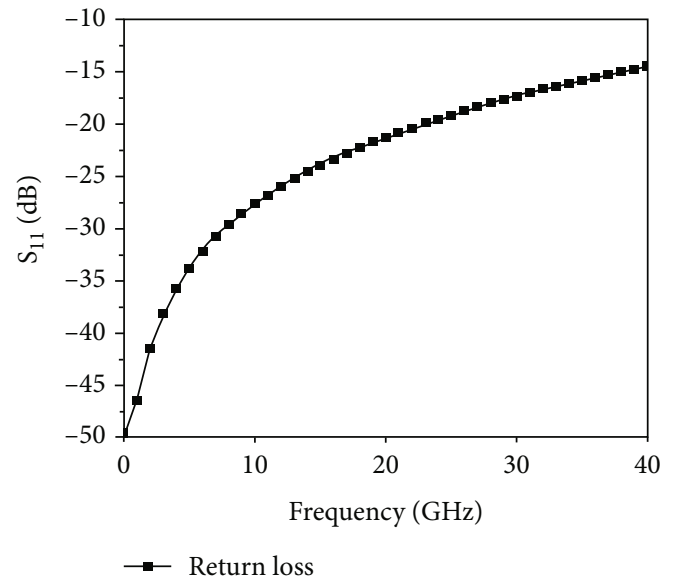

Figure 7: Return loss in upstate as a function of frequency.

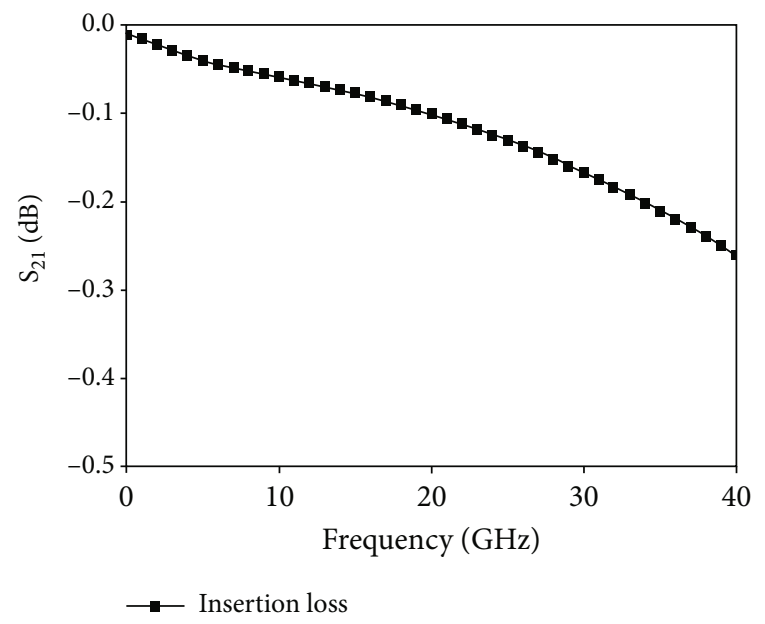

FIGURE 8: Insertion loss in upstate as a function of frequency.

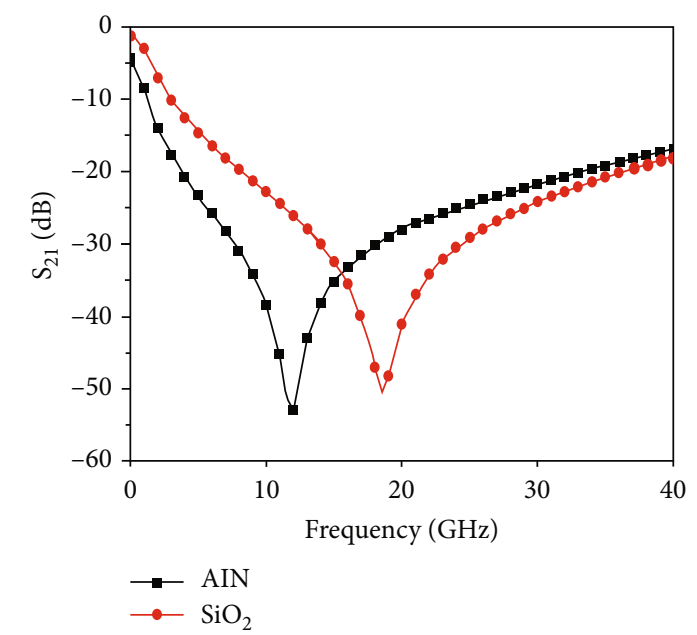

FIGURE 9: Isolation in downstate as a function of frequency.

When the proposed shunt MEMS switch is in an actuated state, switch capacitance is a large increase in downstate by factor 20-100 resulting in the good isolation. Isolation defines

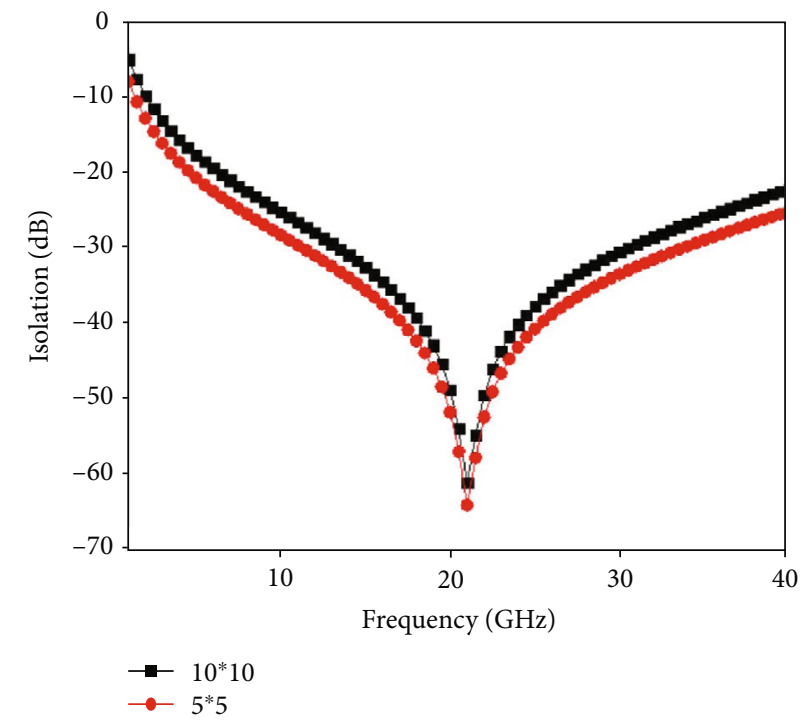

Figure 10: Effect of holes on isolation.

as how much power is present between port 1 and port 2 when the switch is in OFF state and is given as [2].

$$
\left|S_{21}\right|^{2}=\frac{4}{\omega_{0}^{2} Z_{0}^{2} C_{\text {down }}^{2}}
$$

where $C_{\text {down }}$ is the downstate capacitance and is given by [1].

$$
C_{\text {down }}=\frac{\varepsilon_{0} \varepsilon_{r} A}{t_{d}} .
$$

The ratio of capacitance in downstate to upstate is defined as the figure of merit (FOM). The FOM factor for the proposed switch is greater than 50. Figure 9 shows the isolation for the proposed switch is better than $20 \mathrm{~dB}$ for the entire frequency band and maximum isolation is $50 \mathrm{~dB}$ $@ 18 \mathrm{GHz}$ and $55 \mathrm{~dB} @ 12 \mathrm{GHz}$ for $\mathrm{SiO}_{2}$ and AIN dielectric materials, respectively.

In MEMS shunt switch, small diameter holes play the vital role in RF performance as well as in mechanical performance. Minor diameters holes are inserted into the top movable beam to overcome the squeeze air film damping and improve the switching speed of the switch. The total holes area may be $65 \%$ of the whole structure. Therefore, holes discharge some of the residual stress in the membrane and decrease the young modules of the MEMS switch. The effect of holes is negligible in upstate if the holes sizes are less than the 3-5 initial air gap because the fringing field can fill the hole area. In downstate, the holes reduced capacitance as well as isolation of the switch. Figure 10 shows the for holes dimension $5 \times 5 \mu \mathrm{m}^{2}$ isolation is $65 \mathrm{~dB}$ and for $10 \times 10 \mu \mathrm{m}^{2}$ isolation is $60 \mathrm{~dB}$.

\section{Conclusion}

In this work, a detailed analysis of a designed RF MEMS capacitive type shunt switch is outlined. Fixed-fixed beam configuration is chosen here for ease of realization. The 
operating frequency is targeted for the 10 to $40 \mathrm{GHz}$ band. MATLAB is used for all analytical calculation, whereas HFSS and Coventorware are used for the full-wave analysis of the switch structure. The proposed switch shows the pull-in voltage around 9.2 $\mathrm{V}$ for the gold membrane. The RF parameters such as isolation, insertion loss, and return loss are $>20 \mathrm{~dB}$, $>0.5 \mathrm{~dB}$, and $>25 \mathrm{~dB}$, respectively, over the whole frequency band for $\mathrm{SiO}_{2}$ as a dielectric layer. Furthermore, to improve the RF parameters, here, an AIN dielectric material is used instead of $\mathrm{SiO}_{2}$ because of the higher dielectric constant to increase the downstate capacitance and thus improve the isolation. The reported maximum isolation in downstate is $55 \mathrm{~dB}$ at $15 \mathrm{GHz}$.

\section{Data Availability}

Data will be made available on request.

\section{Conflicts of Interest}

The authors declare that there is no conflict of interest regarding the publication of this paper.

\section{References}

[1] G. G. M. Rebeiz, RF MEMS Theory, Design and Technique, Wiley, Hoboken, 2003.

[2] H. Jaafar, K. S. Beh, N. A. Yunus, W. Z. W. Hasan, S. Shafic, and O. Sidek, "A comprehensive study on RF MEMS switch," Microsystem Technologies, vol. 20, no. 12, pp. 2109-2121, 2014.

[3] K. V. Caekenberghe and K. Sarabandi, "A self-aligned fabrication process for capacitive fixed-fixed beam RF MEMS components," Journal of Microelectromechanical Systems, vol. 17, no. 3, pp. 747-754, 2008.

[4] C. Goldsmith, J. Ehmke, A. Malczewski et al., "Lifetime characterization of capacitive Rf MEMS switches," in 2001 IEEE MTT-S International Microwave Sympsoium Digest (Cat. No.01CH37157), pp. 227-230, Phoenix, AZ, USA, May 2001.

[5] C. L. Dai, H. J. Peng, M. C. Liu, C. C. Wu, and L. J. Yang, "Design and fabrication of RF MEMS switch by the CMOS process," Tamkang Journal of Science and Engineering, vol. 8, no. 3, pp. 197-202, 2005.

[6] L. Y. Ma, A. N. Nordin, and N. Soin, "A novel design of a lowvoltage low-loss T-match RF-MEMS capacitive switch," Microsystem Technologies, vol. 24, no. 1, pp. 561-574, 2018.

[7] Y. W. Yu, J. Zhu, S. X. Jia, and Y. Shi, "A high isolation seriesshunt RF MEMS switch," Sensors, vol. 9, no. 6, pp. 4455-4464, 2009.

[8] A. Persano, A. Cola, G. de Angelis, A. Taurino, P. Siciliano, and F. Quaranta, "Capacitive RF MEMS switches with tantalum-based materials," Journal Of Microelectromechanical Systems, vol. 20, no. 2, pp. 365-370, 2011.

[9] W. B. Zheng, Q. A. Huang, X. P. Liao, and F. X. Li, "RF MEMS membrane switches on GaAs substrates for X-band applications," Journal of Microelectromechanical Systems, vol. 14, no. 3, pp. 464-471, 2005.

[10] H. R. Ansari and S. Khosroabadi, "Design and simulation of a novel RF MEMS shunt capacitive switch with a unique spring for Ka-band application," Microsystem Technologies, vol. 25, no. 2, pp. 531-540, 2019.
[11] B. Pillans, J. Kleber, C. Goldsmith, and M. Eberly, "RF power handling of capacitive RF MEMS devices," in 2002 IEEE MTT-S International Microwave Symposium Digest (Cat. No.02CH37278), pp. 329-332, Seattle, WA, USA, June 2002.

[12] A. Persano, A. Tazzoli, A. Cola, P. Siciliano, G. Meneghesso, and F. Quaranta, "Reliability enhancement by suitable actuation waveforms for capacitive RF MEMS switches in III-V technology," Journal of Microelectromechanical Systems, vol. 21, no. 2, pp. 414-419, 2012.

[13] Q. Ma, Q. Tran, T. Kuan et al., Metal Contact Reliability of RF MEMS Switches, Intel Corporation, Santa Clara, CA, USA, 2007.

[14] J. Innnaci, "Reliability of MEMS: a perspective on failure mechanisms, improvement solutions and best practices at development level," Displays, vol. 37, pp. 62-71, 2015.

[15] N. Kobayashi, A. Takano, and S. Kubono, "Measuring equipment and measurements of adhesion force between gold electrical contacts," IEEE Transactions on Components, Packaging and Manufacturing Technology-Part A, vol. 21, no. 1, pp. 46-53, 1998.

[16] M. F. B. Badia, E. Buitrago, and A. M. Ionescu, "RF MEMS shunt capacitive switches using AlN compared to $\mathrm{Si}_{3} \mathrm{~N}_{4}$ dielectric," Journal of Microelectromechanical Systems, vol. 21, no. 5, pp. 1229-1240, 2012.

[17] C. Chu and X. Liao, "One to $40 \mathrm{GHz}$ ultra-wideband RF MEMS direct-contact switch based on GaAs MMIC technique," IET Microwaves, Antennas \& Propagation, vol. 12, no. 6, pp. 879-884, 2017.

[18] A. Ziaei, T. Dean, and Y. Mancuso, "Life time characterization of capacitive power RF MEMS switch," in 2005 European Microwave Conference, Paris, France, October 2005.

[19] J. Y. Park, G. H. Kim, K. W. Chung, and J. U. Bu, "Monolithically integrated micromachined RF MEMS capacitive switches," Sensors and Actuators A: Physical, vol. 89, no. 1-2, pp. 88-94, 2001.

[20] C. L. Goldsmith, Z. Yao, S. Eshelman, and D. Denniston, "Performance of low-loss RF MEMS capacitive switches," IEEE Microwave and Guided Wave Letters, vol. 8, no. 8, pp. 269271, 1998.

[21] S. Fouladi and ,R. R. Mansour, "Capacitive RF MEMS switches fabricated in standard $0.35-\mu \mathrm{m}$ CMOS technology," IEEE Transactions on Microwave Theory and Techniques, vol. 58, no. 2, pp. 478-486, 2010.

[22] K. Jairath, N. Singh, V. Jagota, and M. Shabaz, "Compact ultrawide band metamaterial-inspired split ring resonator structure loaded band notched antenna," Mathematical Problems in Engineering, vol. 2021, Article ID 5174455, 12 pages, 2021.

[23] Z. Deng, H. Wei, S. Fan, and J. Gan, "Design and analysis a novel RF MEMS switched capacitor for low pull-in voltage application," Microsystem Technologies, vol. 22, no. 8, pp. 2141-2149, 2016.

[24] F. M. Guo, Z. Q. Zhu, Y. F. Long et al., "Study on low voltage actuated MEMS rf capacitive switches," Sensors and Actuators A: Physical, vol. 108, no. 1-3, pp. 128-133, 2003. 